\title{
Quadrupole collective dynamics from energy density functionals: Collective Hamiltonian and the interacting boson model
}

\author{
K. Nomura, ${ }^{1,2}$ T. Nikšic, ${ }^{2}$ T. Otsuka, ${ }^{1,3,4}$ N. Shimizu, ${ }^{1,3}$ and D. Vretenarr ${ }^{1,2}$ \\ ${ }^{1}$ Department of Physics, University of Tokyo, Hongo, Bunkyo-ku, Tokyo 113-0033, Japan \\ ${ }^{2}$ Physics Department, Faculty of Science, University of Zagreb, 10000 Zagreb, Croatia \\ ${ }^{3}$ Center for Nuclear Study, University of Tokyo, Hongo, Bunkyo-ku, Tokyo 113-0033, Japan \\ ${ }^{4}$ National Superconducting Cyclotron Laboratory, Michigan State University, East Lansing, Michigan 48824-1321, USA
}

(Received 24 April 2011; revised manuscript received 2 June 2011; published 6 July 2011)

\begin{abstract}
Microscopic energy density functionals have become a standard tool for nuclear structure calculations, providing an accurate global description of nuclear ground states and collective excitations. For spectroscopic applications, this framework has to be extended to account for collective correlations related to restoration of symmetries broken by the static mean field, and for fluctuations of collective variables. In this paper, we compare two approaches to five-dimensional quadrupole dynamics: the collective Hamiltonian for quadrupole vibrations and rotations and the interacting boson model (IBM). The two models are compared in a study of the evolution of nonaxial shapes in Pt isotopes. Starting from the binding energy surfaces of ${ }^{192,194,196} \mathrm{Pt}$, calculated with a microscopic energy density functional, we analyze the resulting low-energy collective spectra obtained from the collective Hamiltonian, and the corresponding IBM Hamiltonian. The calculated excitation spectra and transition probabilities for the ground-state bands and the $\gamma$-vibration bands are compared to the corresponding sequences of experimental states.
\end{abstract}

DOI: 10.1103/PhysRevC.84.014302

PACS number(s): 21.10.Re, 21.60.Ev, 21.60.Fw, 21.60.Jz

\section{INTRODUCTION}

One of the major research topics in theoretical nuclear structure physics has been the study of quadrupole collective dynamics from a microscopic viewpoint [1-4]. Quadrupole collectivity results from multinucleon dynamics of nuclear surface deformation. The equilibrium shape of a nucleus can change depending on the number of valence nucleons: a spherical vibrator, a deformed rotor, or a soft shape in between. In most isotopic or isotonic sequences, the transition between different shapes is gradual, but in a number of cases, with the addition or subtraction of only a few nucleons, one finds signatures of abrupt changes in observables that characterize equilibrium shapes. These structure phenomena have been investigated using concepts of quantum shape-phase transitions [4], and advanced self-consistent beyond-meanfield approaches [5-9].

Microscopic studies based on energy density functionals (EDFs) have been quite successful in reproducing with remarkable accuracy various intrinsic (bulk) properties of medium-mass and heavy nuclei, such as binding energies, density distributions, charge radii, giant resonances, etc. $[2,3]$. The current generation of EDFs includes nonrelativistic Skyrme [10,11] and Gogny [12,13] functionals, as well as relativistic density functionals $[14,15]$. The framework of EDFs has also been extended beyond the mean-field level to describe excitation spectra and electromagnetic transition rates. Models have been developed that perform restoration of symmetries broken by the static nuclear mean field and take into account quadrupole fluctuations: generator coordinate method configuration mixing calculations [2,3,16-18] and solutions of the collective Hamiltonian with quadrupole degrees of freedom $[8,9,19-21]$.
Another successful approach to the low-lying structure of medium-mass and heavy nuclei is based on the interacting boson approximation [22]. The interacting boson model (IBM), in particular, provides not only an algebraic but, in some cases, also a microscopic description of nearly spherical and $\gamma$-unstable shapes [22-27]. From a microscopic point of view, the collective $J=0^{+}$and $2^{+}$pairs of valence protons (neutrons) are mapped onto the corresponding boson images with $J=0^{+}$and $2^{+}$, denoted by $s_{\pi}\left(s_{v}\right)$ and $d_{\pi}\left(d_{v}\right)$ bosons, respectively [23]. A number of studies have been carried out to derive the IBM Hamiltonian, starting from nucleonic degrees of freedom in terms of the conventional mapping method that starts from the shell model [23], the more recent approach based on binding energy maps calculated with microscopic EDFs [26], etc.

A static self-consistent mean-field solution in the intrinsic frame, for instance, a map of the energy surface as a function of quadrupole deformation, is characterized by symmetry breaking - translational, rotational, and particle numberand can only provide an approximate description of bulk ground-state properties. To calculate excitation spectra and electromagnetic transition rates in individual nuclei, it is necessary to include correlations that arise from symmetry restoration and fluctuations around the mean-field minimum. Both types of correlations can be included simultaneously by mixing angular-momentum projected states corresponding to different quadrupole moments. The most effective approach for configuration mixing calculations is the generator coordinate method (GCM), with multipole moments used as coordinates that generate the intrinsic wave functions. It must be noted that, while GCM configuration mixing of axially symmetric states has been implemented by several groups and routinely used in nuclear structure studies [28-30], the 
application of this method to triaxial shapes presents a much more involved and technically difficult problem [18,31]. In addition, the use of general EDFs, that is, with an arbitrary dependence on nucleon densities, in GCM-type calculations, often leads to discontinuities or even divergences of the energy kernels as a function of deformation [32,33]. Only for certain types of density dependence can a regularization method be implemented, which corrects energy kernels and removes the discontinuities and divergences [34-36].

In an approximation to the full GCM approach to fivedimensional quadrupole dynamics that restores rotational symmetry and allows for fluctuations around the triaxial meanfield minima, a collective Hamiltonian can be formulated, with deformation-dependent parameters determined by constrained microscopic self-consistent mean-field calculations. The dynamics of the five-dimensional Hamiltonian for quadrupole vibrational and rotational degrees of freedom is governed by the seven functions of the intrinsic quadrupole deformations: the collective potential, three vibrational mass parameters, and three moments of inertia for rotations around the principal axes [8,9,19-21].

Another approximation consists in mapping the selfconsistent mean-field solution to a boson (IBM) Hamiltonian. In Refs. [26,27], the energy surface for quadrupole degrees of freedom, calculated from a microscopic EDF, was mapped onto the corresponding boson energy surface under certain approximations. The interaction strengths of the boson Hamiltonian are determined by the mapping procedure. One then proceeds to calculate the excitation spectra and wave functions in the laboratory frame [26,27]. The validity of the method of Ref. [26] was tested in various mass regions $[27,37,38]$.

It would, therefore, be interesting to compare the two approximations starting from the same self-consistent meanfield solution based on a microscopic EDF. In this paper, we compare spectroscopic observables calculated with the IBM Hamiltonian to the solution of the collective quadrupole Hamiltonian, with both calculations based on relativistic Hartree-Bogoliubov (RHB) [14] self-consistent binding energy surfaces. The framework of relativistic EDFs and the corresponding collective Hamiltonian have successfully been employed in studies of the evolution of ground-state shapes and spectroscopic properties of medium-heavy and heavy nuclei $[8,9,15,21,39]$. In the present analysis, we consider the even-even isotopes ${ }^{192-196} \mathrm{Pt}$. In the IBM framework, these $\gamma$-soft nuclei can be characterized by the $\mathrm{O}(6)$ dynamical symmetry $[22,40,41]$.

The paper is organized as follows. In Sec. II, we briefly describe the theoretical procedures used to derive the collective Hamiltonian and the IBM Hamiltonian starting from a given EDF. The microscopic RHB energy surface and the mapped IBM energy surface are discussed in Sec. III. Spectroscopic properties of ${ }^{192-196} \mathrm{Pt}$ calculated with the two models are compared in Sec. IV. Section V summarizes the results and presents a short outline of future work.

\section{THEORETICAL FRAMEWORK}

The map of the energy surface as a function of the quadrupole collective variables $\beta$ and $\gamma$ [1] is obtained from self-consistent RHB calculations with additional constraints on the axial and triaxial mass quadrupole moments. The quadrupole moments can be related to the polar deformation parameters $\beta$ and $\gamma$. The parameter $\beta$ is simply proportional to the intrinsic quadrupole moment, and the angular variable $\gamma$ specifies the type and orientation of the shape. The limit $\gamma=0$ corresponds to axial prolate shapes, whereas the shape is oblate for $\gamma=\pi / 3$. Triaxial shapes are associated with intermediate values $0<\gamma<\pi / 3$. In this paper, the constrained RHB calculations have been performed using the relativistic functional DD-PC1 [42]. Starting from microscopic nucleon self-energies in nuclear matter, and empirical global properties of the nuclear matter equation of state, the coupling parameters of DD-PC1 have been determined in a careful comparison of the calculated binding energies with data, for a set of 64 axially deformed nuclei in the mass regions $A \approx$ 150-180 and $A \approx 230-250$. DD-PC1 has been further tested in a series of calculations of properties of spherical and deformed medium-heavy and heavy nuclei, including binding energies, charge radii, deformation parameters, neutron skin thickness, and excitation energies of giant multipole resonances. For the examples presented here, pairing correlations have been taken into account by employing a pairing force that is separable in momentum space, and is completely determined by two parameters adjusted to reproduce the empirical bell-shaped pairing gap in symmetric nuclear matter [43].

The entire dynamics of the collective Hamiltonian is governed by seven functions of the intrinsic deformations $\beta$ and $\gamma$ : the collective potential, the three mass parameters: $B_{\beta \beta}$, $B_{\beta \gamma}, B_{\gamma \gamma}$, and the three moments of inertia $\mathcal{I}_{k}$. These functions are determined by the choice of a particular microscopic nuclear energy density functional and a pairing functional. The quasiparticle wave functions and energies, that correspond to constrained self-consistent solutions of the RHB model, provide the microscopic input for the parameters of the collective Hamiltonian [21],

$$
\hat{H}_{\text {coll }}=\hat{T}_{\text {vib }}+\hat{T}_{\text {rot }}+V_{\text {coll }},
$$

with the vibrational kinetic energy,

$$
\begin{aligned}
\hat{T}_{\mathrm{vib}}= & -\frac{\hbar^{2}}{2 \sqrt{w r}}\left\{\frac { 1 } { \beta ^ { 4 } } \left[\frac{\partial}{\partial \beta} \sqrt{\frac{r}{w}} \beta^{4} B_{\gamma \gamma} \frac{\partial}{\partial \beta}\right.\right. \\
& \left.-\frac{\partial}{\partial \beta} \sqrt{\frac{r}{w}} \beta^{3} B_{\beta \gamma} \frac{\partial}{\partial \gamma}\right]+\frac{1}{\beta \sin 3 \gamma}\left[-\frac{\partial}{\partial \gamma} \sqrt{\frac{r}{w}} \sin 3 \gamma\right. \\
& \left.\left.\times B_{\beta \gamma} \frac{\partial}{\partial \beta}+\frac{1}{\beta} \frac{\partial}{\partial \gamma} \sqrt{\frac{r}{w}} \sin 3 \gamma B_{\beta \beta} \frac{\partial}{\partial \gamma}\right]\right\}
\end{aligned}
$$

and rotational kinetic energy,

$$
\hat{T}_{\text {rot }}=\frac{1}{2} \sum_{k=1}^{3} \frac{\hat{J}_{k}^{2}}{\mathcal{I}_{k}} .
$$

$V_{\text {coll }}$ is the collective potential. $\hat{J}_{k}$ denotes the components of the angular momentum in the body-fixed frame of a nucleus, and the mass parameters $B_{\beta \beta}, B_{\beta \gamma}, B_{\gamma \gamma}$, as well as the moments of inertia $\mathcal{I}_{k}$, depend on the quadrupole deformation variables $\beta$ and $\gamma: \mathcal{I}_{k}=4 B_{k} \beta^{2} \sin ^{2}(\gamma-2 k \pi / 3)$. Two additional quantities that appear in the expression for the vibrational energy, $r=B_{1} B_{2} B_{3}$ and $w=B_{\beta \beta} B_{\gamma \gamma}-B_{\beta \gamma}^{2}$, 
determine the volume element in the collective space. The moments of inertia are computed using the Inglis-Belyaev (IB) formula $[44,45]$, and the mass parameters associated with the two quadrupole collective coordinates $q_{0}=\left\langle\hat{Q}_{20}\right\rangle$ and $q_{2}=\left\langle\hat{Q}_{22}\right\rangle$ are calculated in the cranking approximation. The potential $V_{\text {coll }}$ in the collective Hamiltonian Eq. (1) is obtained by subtracting the zero-point energy corrections from the total energy that corresponds to the solution of constrained RHB equations, at each point on the triaxial deformation plane. The Hamiltonian Eq. (1) describes quadrupole vibrations, rotations, and the coupling of these collective modes. The corresponding eigenvalue problem is solved using an expansion of eigenfunctions in terms of a complete set of basis functions that depend on the deformation variables $\beta$ and $\gamma$, and the Euler angles $\phi, \theta$, and $\psi$ [21]. The diagonalization of the Hamiltonian yields the excitation energies and collective wave functions for each value of the total angular momentum and parity that are used to calculate observables. An important advantage of using the collective model based on self-consistent meanfield single-(quasi)particle solutions is the fact that physical observables, such as transition probabilities and spectroscopic quadrupole moments, are calculated in the full configuration space and there is no need for effective charges. Using the bare value of the proton charge in the electric quadrupole operator, the transition probabilities between eigenvectors of the collective Hamiltonian can be directly compared with data.

In an equivalent approach, the RHB binding energy surface can be mapped onto the IBM Hamiltonian. Starting from the energy surface $E_{\mathrm{RHB}}(\beta, \gamma)$ calculated with the DD-PC1 plus separable-pairing functional, each point on the $(\beta, \gamma)$ plane is mapped onto the corresponding point on the energy surface calculated in the IBM, referred to hereafter as $E_{\mathrm{IBM}}\left(\beta_{B}, \gamma_{B}\right)$, using the method proposed in Ref. [27]. Here $\beta_{B}$ and $\gamma_{B}$ denote the boson images of the quadrupole deformation parameters $\beta$ and $\gamma$, respectively, that are used as constraints in the self-consistent RHB calculation and appear as variables in the collective Hamiltonian. The boson images $\beta_{B}$ and $\gamma_{B}$ are related to $\beta$ and $\gamma$ through the proportionality $\beta_{B} \propto \beta$, and the equality $\gamma_{B}=\gamma$, respectively [26,27]. This mapping procedure is used to determine the strength parameters of the IBM Hamiltonian.

We consider the proton-neutron IBM (IBM-2) model [23]: the number of proton (neutron) bosons, denoted by $n_{\pi}\left(n_{v}\right)$, are assumed to equal half the number of valence protons (neutrons). In the consistent- $Q$ formalism [22] the IBM-2 Hamiltonian reads

$$
\hat{H}_{\mathrm{IBM}}=\epsilon\left(\hat{n}_{d \pi}+\hat{n}_{d \nu}\right)+\kappa \hat{Q}_{\pi} \cdot \hat{Q}_{\nu},
$$

where $\hat{n}_{d \rho}=d_{\rho}^{\dagger} \cdot \tilde{d}_{\rho} \quad(\rho=\pi \quad$ or $\quad v)$ and $\hat{Q}_{\rho}=\left[s_{\rho}^{\dagger} \tilde{d}_{\rho}+\right.$ $\left.d_{\rho}^{\dagger} \tilde{s}_{\rho}\right]^{(2)}+\chi_{\rho}\left[d_{\rho}^{\dagger} \tilde{d}_{\rho}\right]^{(2)}$ denote the $d$-boson number operator and the quadrupole operator, respectively. $\epsilon$ and $\kappa$ are coupling constants. The parameters $\chi_{\pi, v}$ inside the quadrupole operators are quite relevant to determining whether a nucleus is prolate or oblate deformed.

The bosonic energy surface $E_{\mathrm{IBM}}(\beta, \gamma)$ corresponds to the classical limit of the Hamiltonian $\hat{H}_{\mathrm{IBM}}: E_{\mathrm{IBM}}\left(\beta_{B}, \gamma_{B}\right)=$ $\left\langle\Psi\left(\beta_{B}, \gamma_{B}\right)\left|\hat{H}_{\mathrm{IBM}}\right| \Psi\left(\beta_{B}, \gamma_{B}\right)\right\rangle . \quad\left|\Psi\left(\beta_{B}, \gamma_{B}\right)\right\rangle$ denotes the boson coherent state [46]: $\left|\Psi\left(\beta_{B}, \gamma_{B}\right)\right\rangle \propto \prod_{\rho=\pi, v}\left[s_{\rho}^{\dagger}+\right.$ $\left.\beta_{\rho} \cos \gamma_{\rho} d_{\rho 0}^{\dagger}+\frac{1}{\sqrt{2}} \beta_{\rho} \sin \gamma_{\rho}\left(d_{\rho+2}^{\dagger}+d_{\rho-2}^{\dagger}\right)\right]^{n_{\rho}}|0\rangle$, up to a normalization constant. Here $|0\rangle$ is the boson vacuum and the variables $\beta_{\rho}$ and $\gamma_{\rho}$ are the corresponding polar deformation parameters. As in our previous studies [26,27], it is assumed that $\beta_{\pi}=\beta_{v} \equiv \beta_{B}$ and $\gamma_{\pi}=\gamma_{\nu} \equiv \gamma_{B}$. The analytical form of $E_{\mathrm{IBM}}\left(\beta_{B}, \gamma_{B}\right)$ can be found in Refs. [26,27]. Hereafter, we denote the bosonic energy surface as $E_{\mathrm{IBM}}(\beta, \gamma)$, omitting the indices of $\beta_{B}$ and $\gamma_{B}$.

The boson Hamiltonian $\hat{H}_{\mathrm{IBM}}$, parametrized by the microscopically calculated coupling constants, is diagonalized in the $M=0$ boson space. Here $M$ denotes the $z$ component of the total boson angular momentum $L$. Reduced quadrupole transition probabilities $B(\mathrm{E} 2)$ are calculated for transitions between the eigenstates of the IBM Hamiltonian.

Here we point out again that the total boson energy $E_{\mathrm{IBM}}(\beta, \gamma)$ has been related to the microscopic EDF energy surface (total energy). However, for the IBM Hamiltonian $\hat{H}_{\text {IBM }}$, one cannot make a distinction between the kinetic and potential terms, as in the corresponding collective Hamiltonian $\hat{H}_{\text {coll }}$. Nevertheless, the effects relevant to both vibrational and rotational kinetic energies are assumed to be incorporated into the IBM approach by adjusting $E_{\mathrm{IBM}}(\beta, \gamma)$ to be as close as possible to the microscopic surface $E_{\mathrm{RHB}}(\beta, \gamma)$. This prescription turned out to be valid for vibrational and $\gamma$-soft nuclei at moderate quadrupole deformation [26,27], similarly to the conventional mapping method of Ref. [23]. For rotational nuclei with large quadrupole deformation, however, the overall scale of the IBM rotational spectra differs from the experimental one [26,27]. The discrepancy partially arises because nuclear rotational properties, characterized by the overlap of the intrinsic state and the rotated one, differ from the rotational characteristics of the corresponding boson system [47]. This problem may be cured by the recently proposed prescription [47], in which the rotational response (i.e., cranking) of the boson system is related to the rotational response of the nucleon system. This procedure goes beyond simple analysis of the zero-frequency energy surface. In order that the boson rotational response becomes equal to the fermion (nucleon) response, an additional kinetic term $\hat{L} \cdot \hat{L}$ has to be included in the boson Hamiltonian, with a coupling constant determined microscopically [47]. The term $\hat{L} \cdot \hat{L}$ directly influences the moment of inertia of rotational band with the eigenvalue $L(L+1)$. However, the above-mentioned problem, concerning the IBM rotational spectra, does not occur in the considered Pt nuclei, and thus one does not need to include the $\hat{L} \cdot \hat{L}$ term in the present case.

Similar problems with the overall scale of the rotational spectra are also encountered in the collective Hamiltonian model, when the IB formula is used to calculate the moments of inertia $[8,9,21]$. The inclusion of an additional scale parameter is often necessary because of the well-known fact that the IB formula predicts effective moments of inertia that are considerably smaller than empirical values. More realistic values are only obtained if one uses the Thouless-Valatin (TV) formula, but this procedure is computationally much more demanding. In the present case, we have used the IB moments of inertia in the calculation of excitation spectra of Pt nuclei, and the agreement with experiment is such that no 
renormalization of the effective moments of inertia is required. This result allows for a direct comparison of the IBM spectra to the solutions of the collective Hamiltonian.

\section{BINDING ENERGY SURFACES IN THE $\beta-\gamma$ PLANE}

Most deformed nuclei display axially symmetric prolate ground-state shapes, but few areas of the nuclide chart are characterized by the occurrence of nonaxial shapes. One example is the $A \approx 190$ mass region, where both prolate to oblate shape transitions, and even triaxial ground-state shapes, have been predicted.

The left-hand side of Fig. 1 shows the self-consistent RHB quadrupole binding energy maps of the ${ }^{192,194,196} \mathrm{Pt}$ isotopes in the $\beta-\gamma$ plane, calculated with the DD-PC1 energy density functional. The energy surfaces are $\gamma$ soft, with shallow minima at $\gamma \approx 30^{\circ}$. In general, the equilibrium deformation decreases with mass number and, proceeding to even heavier isotopes, one finds that the energy map of ${ }^{198} \mathrm{Pt}$ has also a nonaxial minimum, whereas ${ }^{200} \mathrm{Pt}$ displays a slightly oblate minimum [15], signaling the shell closure at the neutron number $N=126$. On the right-hand side of Fig. 1, we plot the corresponding IBM energy surfaces $E_{\mathrm{IBM}}(\beta, \gamma)$, obtained by mapping each point of surface $E_{\mathrm{RHB}}(\beta, \gamma)$ onto the energy surface calculated in the IBM, following the procedure of Ref. [27]. To be able to compare the low-energy spectra in the two models, the IBM surfaces are mapped in such a way as to reproduce the RHB energy surfaces up to $\approx 2 \mathrm{MeV}$ above the mean-field minimum. This means that the maps shown in Fig. 1 can only be compared for values of $\beta$ not very different from the minimum $\beta_{\min }$. For larger values of $\beta$, that is, for higher excitation energies, the topology of the RHB surfaces is determined by single-nucleon configurations that are not included in the model space (valence space) from which the IBM bosons are constructed. For large $\beta$ deformations, therefore, one should not try to map the microscopic energy surfaces onto the IBM. This is the reason why the IBM energy surfaces are, by construction, always rather flat in the region $\beta \gg \beta_{\min }$. In the vicinity of the minima, the curvatures of the IBM energy maps are rather similar to those of the original RHB surfaces both in $\beta$ and $\gamma$ directions. The derived values for the $\chi_{\pi}$ and $\chi_{\nu}$ parameters in Eq. (4) satisfy $\chi_{\pi}+\chi_{\nu} \sim 0$, characteristic for a $\gamma$-soft energy surface.

One might notice that the IBM energy maps reproduce the value of $\beta$ at the minima predicted by the RHB calculation, whereas the mapping does not reproduce the shallow triaxial minima of the RHB surfaces. The minima of the IBM maps are either oblate or prolate. This is because the IBM Hamiltonian of Eq. (4) is too restricted to produce a triaxial minimum. In the analytical expression for $E_{\mathrm{IBM}}(\beta, \gamma)$, the $\gamma$-dependent term is proportional to $\left(\chi_{\pi}+\chi_{\nu}\right) \cos 3 \gamma$, and this places the minimum either on the prolate or oblate side according to the sign of $\left(\chi_{\pi}+\chi_{\nu}\right)$. The Pt nuclei considered here do not display any rapid structural change but remain $\gamma$ soft. This feature appears to be independent of the choice of the EDF. A recent microscopic calculation using the Gogny-D1S EDF [13] also yielded shallow triaxial shapes, rather flat in the oblate region [37], but quantitatively consistent with the present analysis. A similar trend was reported in other EDF-based studies of ground-state shapes of Pt isotopes [39,48,49]. In the present calculation, the RHB surfaces become softer in $\gamma$ with increasing neutron number, and the softest nucleus is ${ }^{196} \mathrm{Pt}$. The corresponding IBM energy surfaces follow this evolution, but do not reproduce the triaxial minima because of the reasons explained above. The recent Gogny-EDF calculation [37] predicts ${ }^{192} \mathrm{Pt}$ to be the softest $\mathrm{Pt}$ isotope in this mass region.

\section{SPECTROSCOPIC PROPERTIES}

In Fig. 2, we display the corresponding low-energy collective spectra of ${ }^{192,194,196} \mathrm{Pt}$ obtained from the collective Hamiltonian (middle panels) and the IBM Hamiltonian (panels on the right). The calculated ground-state and (quasi-) $\gamma$ vibration bands are compared to the corresponding sequences of experimental states [50]. The eigenstates of the collective Hamiltonian in Eq. (1) are completely determined by the DD-PC1 energy density functional plus a separable pairing interaction, and the transition probabilities are calculated in the full configuration space using the bare value of the proton charge. Since $\hat{H}_{\mathrm{IBM}}$ in Eq. (4) acts only in the boson valence space, to calculate the $B(\mathrm{E} 2)$ values, one needs two additional parameters: the proton-boson and neutron-boson effective charges. For simplicity, here we take these effective charges to be equal, and in each nucleus normalize the $B(\mathrm{E} 2)$ values obtained in the IBM to reproduce the transition probability $B\left(\mathrm{E} 2 ; 2_{1}^{+} \rightarrow 0_{1}^{+}\right)$calculated with the collective Hamiltonian. Thus we can only compare the ratios of the IBM $B(\mathrm{E} 2)$ values, divided by $B\left(\mathrm{E} 2 ; 2_{1}^{+} \rightarrow 0_{1}^{+}\right)$, to those predicted by the collective Hamiltonian based on DD-PC1, and to available data.

For the ground-state band, both the collective Hamiltonian and the IBM predict excitation spectra in close agreement with experiment. For ${ }^{192} \mathrm{Pt}$, in particular, the calculated ground-state bands seem to indicate a somewhat larger deformation than observed experimentally. In fact, the theoretical energy ratio $R_{4 / 2}=E\left(4_{1}^{+}\right) / E\left(2_{1}^{+}\right)$is 2.59 with the collective Hamiltonian and 2.69 with the IBM Hamiltonian, compared to the experimental value $R_{4 / 2}=2.48$. A similar trend is also found for the other two nuclei. A more pronounced difference between the predictions of the two models is found in the E2 decay pattern of the ground-state band, particularly in ${ }^{194} \mathrm{Pt}$ nucleus for which data are available up to angular momentum $10^{+}$. For the spectrum calculated with the collective model, the E2 transition rates from the state with angular momentum $L$ ( $L \geqslant 2$ ) to the one with $L-2$ keep increasing as functions of $L$, even though the corresponding experimental $B(E 2)$ values in ${ }^{192,194} \mathrm{Pt}$ decrease starting from $L=6$. The trend of the $B$ (E2) values calculated with the IBM, on the other hand, is much closer to experiment. The $B(\mathrm{E} 2)$ 's decrease in the IBM because the model space is built from valence nucleons only, and the wave functions of higher angular-momentum states correspond to simple configurations of fully aligned $d$ bosons [22], whereas there is no limit on the angular momentum of eigenstates of the collective Hamiltonian.

A more significant difference between the spectroscopic properties predicted by the collective Hamiltonian and the IBM 

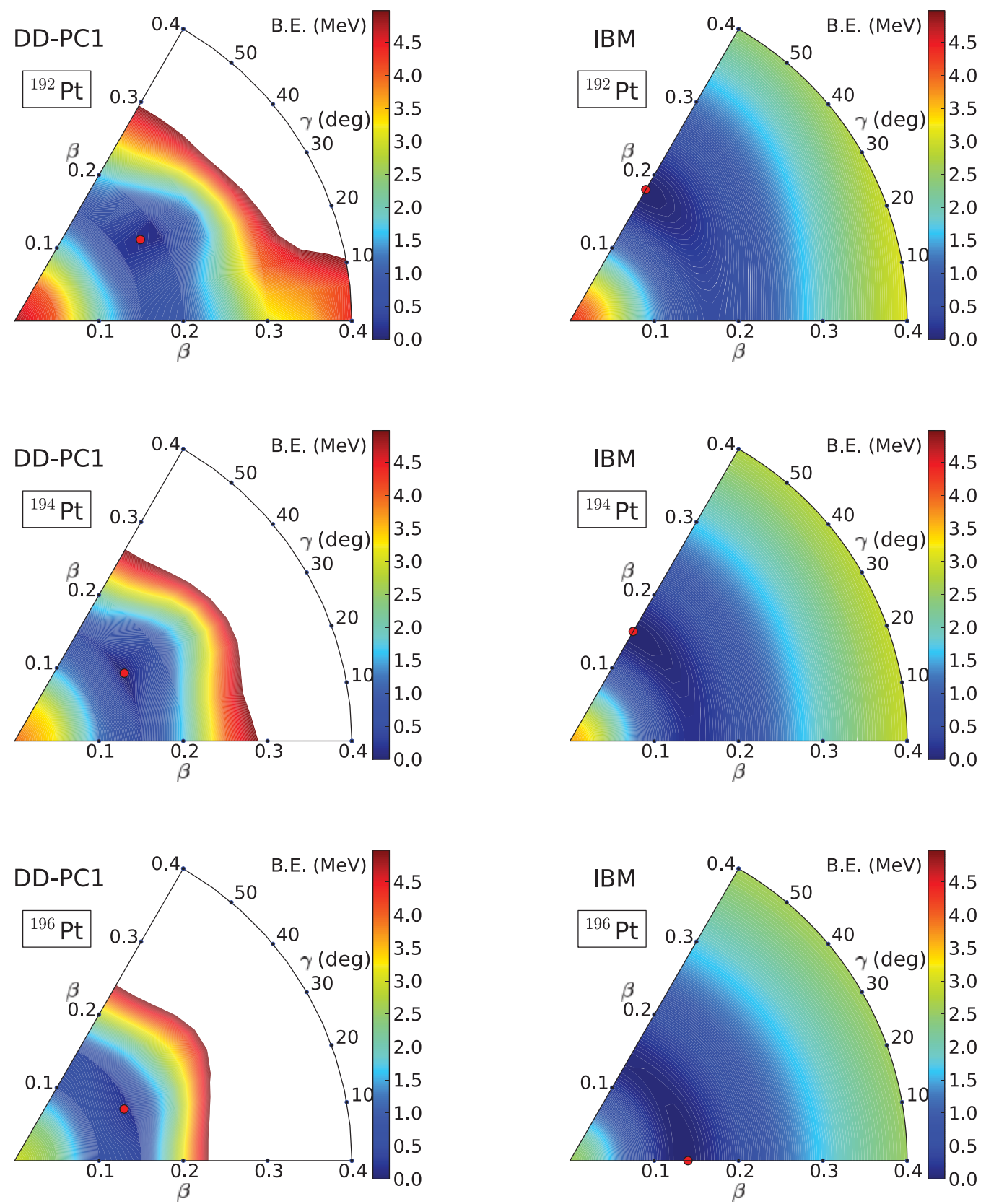

FIG. 1. (Color online) Self-consistent binding-energy maps of ${ }^{192,194,196} \mathrm{Pt}$ in the $\beta$ - $\gamma$ plane $\left(0^{\circ} \leqslant \gamma \leqslant 60^{\circ}\right)$, calculated with the RHB model using the DD-PC1 functional (left panels), and the corresponding mapped energy surface of the IBM, $E_{\mathrm{IBM}}\left(\beta_{B}, \gamma_{B}\right)$. The IBM total energies are depicted in terms of $\beta$ and $\gamma$, where $\beta \propto \beta_{B}$ and $\gamma=\gamma_{B}$ (see text for definition).

is found in the sequence of levels built on the state $2_{2}^{+}$-the (quasi-) $\gamma$ band. The IBM spectra display a staggering of excitation energies above $2_{\gamma}^{+}$, with the formation of doublets $\left(3_{\gamma}^{+} 4_{\gamma}^{+}\right),\left(5_{\gamma}^{+} 6_{\gamma}^{+}\right)$, etc., whereas the collective Hamiltonian yields a regular excitation pattern consistent with the experimental band. To be more precise, the IBM spectra correspond to $\gamma$-unstable nuclei, and are close to the limit of $\mathrm{O}(6)$ dynamical symmetry, in which eigenstates of a boson Hamiltonian with the same $\tau$ quantum number are degenerate [40]. On the other hand, the $\gamma$ bands predicted by the collective model, as well as the experimental sequence, seem to be closer to rigid triaxiality [52]. The difference between the collective Hamiltonian and the IBM arises probably because the shallow triaxial minima of the RHB energy surfaces are not reproduced by the mapping onto the IBM total energy (cf. Fig. 1). The agreement of the IBM (quasi-) $\gamma$ band with experiment could be improved by introducing additional interaction terms in the IBM Hamiltonian, i.e., three-body terms (the so-called cubic terms) [53,54]. Terms of this type will have to be included for a more precise analysis and comparison of states above the yrast with experimental results.

A nice feature of the present calculation, particularly the one with the IBM Hamiltonian, is that the predicted 

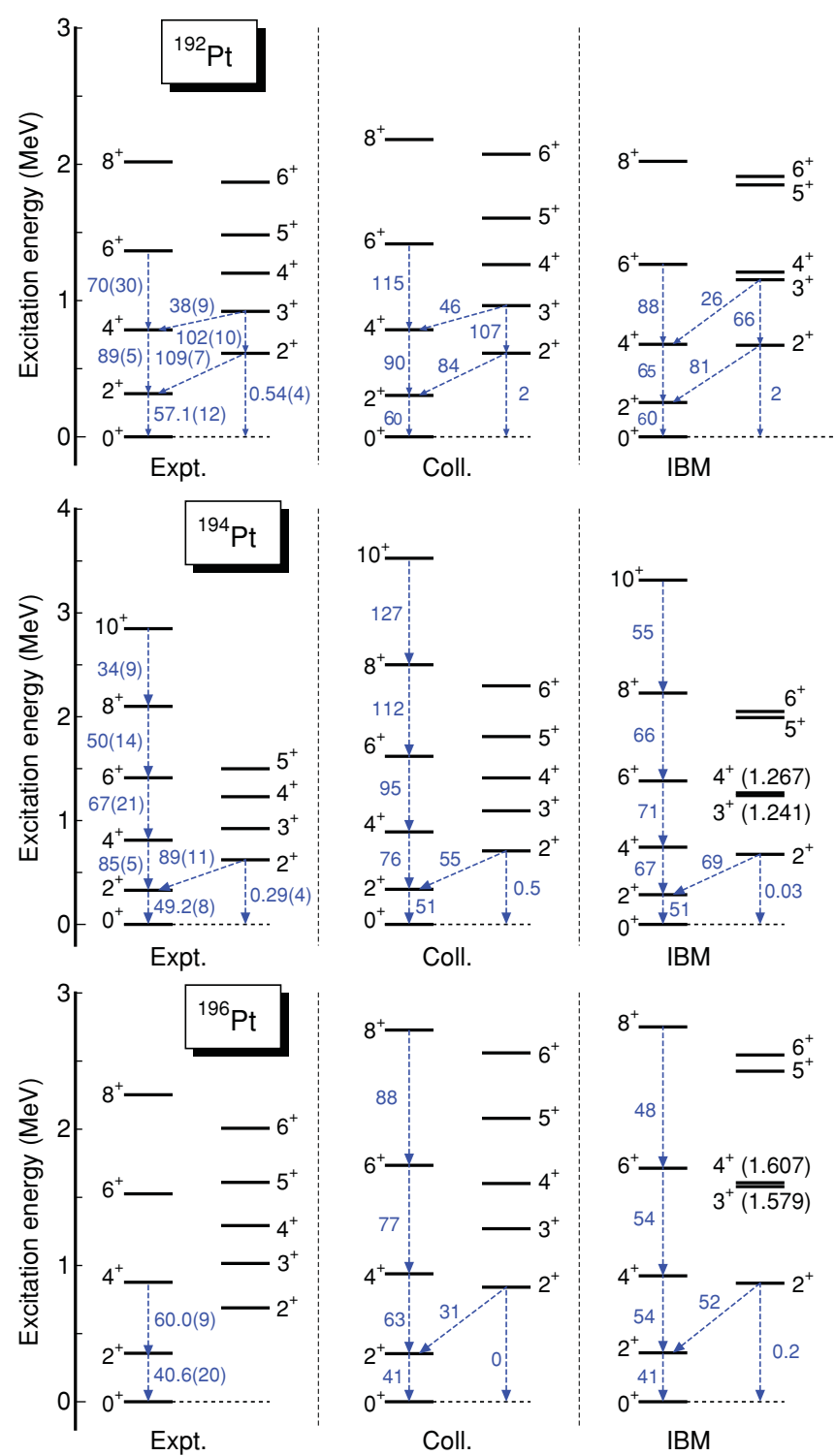

FIG. 2. (Color online) Low-lying collective spectra of ${ }^{192,194,196} \mathrm{Pt}$ nuclei, calculated with the collective Hamiltonian based on the DD-PC1 functional and the corresponding IBM Hamiltonian, in comparison with available data. For each nucleus, the $B(\mathrm{E} 2)$ values (in Weisskopf units) obtained in the IBM are normalized to the $B\left(\mathrm{E} 2 ; 2_{1}^{+} \rightarrow 0_{1}^{+}\right)$predicted by the collective Hamiltonian. The experimental excitation spectra and $B(\mathrm{E} 2)$ values are from Refs. [50] and [51], respectively.

$B$ (E2) values for the transition $2_{2}^{+} \rightarrow 2_{1}^{+}$are comparable to or even larger than those corresponding to $4_{1}^{+} \rightarrow 2_{1}^{+}$. This result is consistent with the experimental trend, whereas in the recent Gogny-based EDF calculation of Ref. [37], the $2_{2}^{+} \rightarrow 2_{1}^{+}$transitions were much weaker than $4_{1}^{+} \rightarrow 2_{1}^{+}$. The corresponding Gogny energy surfaces displayed pronounced oblate minima in Ref. [37], unlike the present energy maps shown in Fig. 1.

Finally, in Figs. 3 and 4, we compare the absolute squares of the collective wave functions for the yrast states $0_{1}^{+}, 2_{1}^{+}$, and $4_{1}^{+}$, and the bandhead of the $\gamma$ band of ${ }^{192} \mathrm{Pt}$, calculated in the two models. These quantities are proportional to the probability density distributions in the $\beta-\gamma$ plane. Figure 3 shows the distributions $\sum_{M=-L}^{L}\left|\left\langle\Phi_{M}^{L} \mid \Psi(\beta, \gamma)\right\rangle\right|^{2}$, where $\left|\Phi_{M}^{L}\right\rangle$ denotes the IBM eigenstate for the state with angular momentum $L$ and projection $M$. The wave functions of the yrast states are concentrated along the oblate axis, only for the state $4_{1}^{+}$the maximum of the absolute square is located at $\gamma \sim 55^{\circ}$, and somewhat larger deviations from pure oblate configurations are found for higher angular momenta. For the state $2_{2}^{+}$, on the other hand, the peak appears in the triaxial region $\left(\gamma \sim 35^{\circ}\right)$, and the distribution is extended more toward oblate quadrupole deformations. The rather large overlap of the collective wave functions for the states $2_{1}^{+}$and $2_{2}^{+}$explains the particularly strong $2_{2}^{+} \rightarrow 2_{1}^{+}$transitions in this nucleus, and similarly in the other two Pt isotopes considered here. The corresponding absolute squares of the eigenstates of the collective Hamiltonian are shown in Fig. 4. In this case, already the wave functions of the yrast states reflect the $\gamma$ softness of the RHB energy surface, and the maxima of the absolute squares are found in the triaxial region of the $\beta-\gamma$ plane.

\section{CONCLUSIONS AND OUTLOOK}

Structure phenomena related to shape evolution currently present a very active research field in low-energy nuclear physics. Radioactive-beam facilities continue to provide interesting new data on shapes in regions of exotic nuclei far from stability. The variation of ground-state shapes is, of course, governed by the evolution of the underlying shell structure of single-nucleon orbitals. It is, therefore, important to develop microscopic approaches that can be employed in quantitative analyses of shape phenomena and the resulting complex excitation spectra and decay patterns across the entire chart of nuclides. Such a framework is provided by nuclear energy density functionals (EDFs).

The advantages of EDFs are evident already at the basic level of implementation: an intuitive interpretation of selfconsistent mean-field results in terms of intrinsic shapes and single-particle states, and the universality of EDFs that enables their applications to all nuclei throughout the periodic chart. The latter is particularly important for extrapolations to regions of short-lived nuclei far from stability. When considering spectroscopic applications, the framework of EDF must be extended beyond the mean-field level to allow for a systematic treatment of dynamical effects related to restoration of broken symmetries and fluctuations in collective coordinates. To calculate excitation spectra and transition rates, it is necessary to project states with good quantum numbers from the meanfield solution, and also take into account fluctuations around the mean-field minimum.

In this work, we have compared two well-known models that explicitly consider quadrupole collective correlations, both starting from maps of binding energy calculated with the same microscopic EDF. The first is the generalized collective Hamiltonian for quadrupole vibrations and rotations. The dynamics of the five-dimensional Hamiltonian is governed by the collective potential, the three vibrational mass parameters, and three moments of inertia for rotations around the principal 

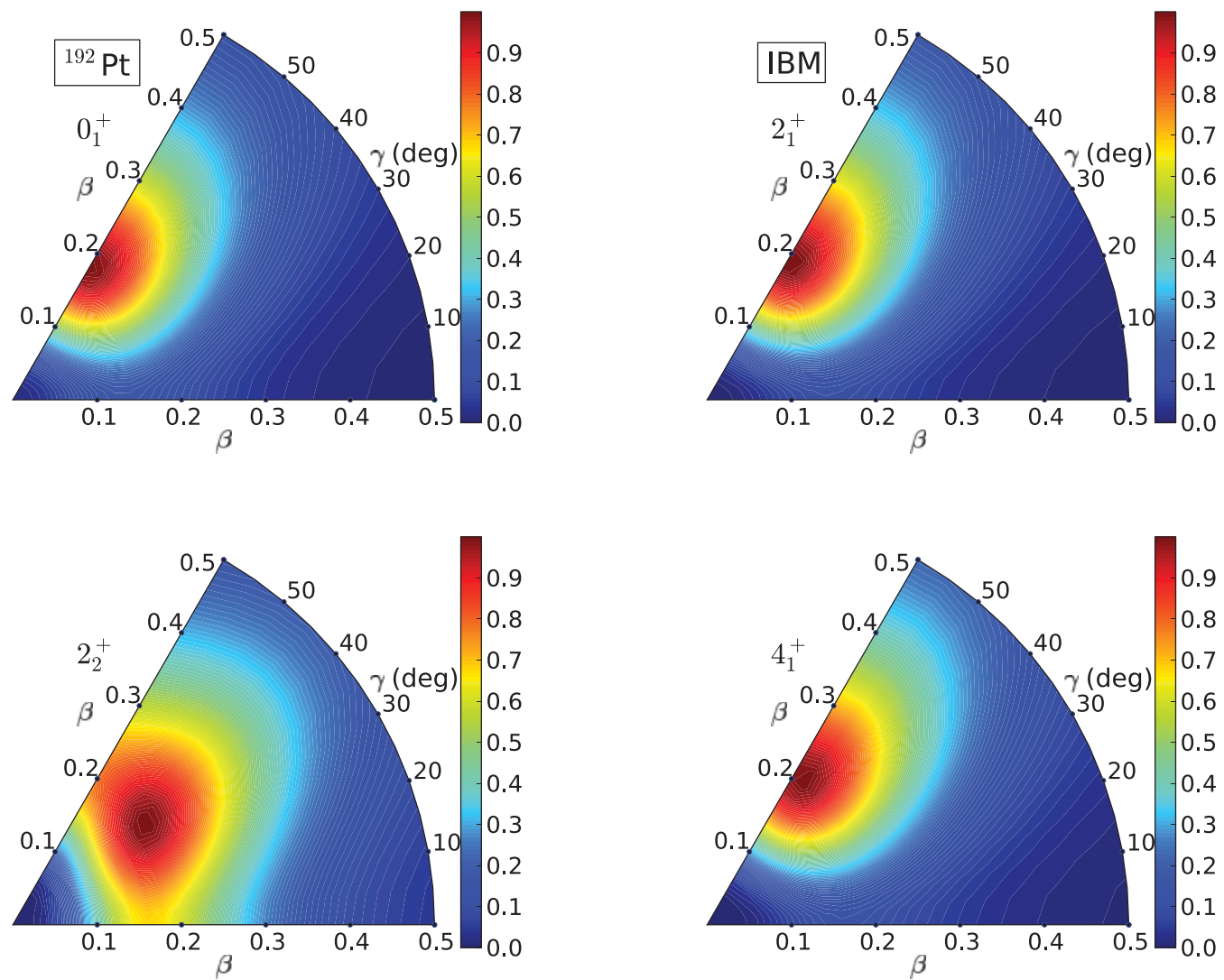

FIG. 3. (Color online) Absolute squares of the IBM wave functions in the $\beta-\gamma$ plane for the yrast states $0_{1}^{+}, 2_{1}^{+}$, and $4_{1}^{+}$, and the bandhead of the $\gamma$ band $2_{2}^{+}$of ${ }^{192} \mathrm{Pt}$.

axes. These functions of the quadrupole deformation parameters are determined by constrained mean-field calculations using a given nuclear EDF. The diagonalization of the resulting Hamiltonian yields excitation energies and collective wave functions that can be used to calculate various observables. Calculations are performed in the full model space of occupied states (no distinction between core and valence nucleons and no need for effective charges). The second model considered in this work is the well-known and very successful IBM-2. In this approach, the configuration space is first restricted to valence nucleons only, and further mapped to the space of $s$ and $d$ bosons. To determine the parameters of the IBM Hamiltonian, the energy surface calculated using a microscopic EDF is mapped onto the corresponding boson energy surface under certain approximations. One then proceeds to calculate the excitation spectra and wave functions in the laboratory frame. To calculate transition probabilities, however, one needs to adjust the effective boson charges. Here this has been done so that, in each nucleus, the calculated $B\left(\mathrm{E} 2 ; 2_{1}^{+} \rightarrow\right.$ $0_{1}^{+}$) coincides with the value obtained using the collective Hamiltonian.

The two models have been compared here in a study of the evolution of nonaxial shapes in Pt isotopes. Starting from the binding energy surfaces of ${ }^{192,194,196} \mathrm{Pt}$, calculated with the DD-PC1 energy density functional plus a separable pairing interaction, we have analyzed the resulting low-energy collective spectra obtained from the collective Hamiltonian and the corresponding IBM-2 Hamiltonian. The calculated ground-state and $\gamma$-vibration bands have also been compared to the corresponding sequences of experimental states. Both models predict that excitation energies and $B(\mathrm{E} 2)$ values are in agreement with data. In particular, we notice the excellent result for the predicted excitation energy of the bandhead of the $\gamma$ band, as well as the good agreement with the experimental $B$ (E2) values for transitions between the $\gamma$ band and the yrast band.

There are also significant differences in the predictions of the two models. With the present form of the IBM Hamiltonian, restricted to two-body boson interactions, its expectation value in the boson coherent state does not reproduce the shallow triaxial minima of the binding energy maps predicted by the constrained self-consistent mean-field calculation using DDPC1. Since the mapped IBM energy surface is $\gamma$ soft rather than triaxial, the resulting spectra display a staggering of excitation energies above $2_{\gamma}^{+}$, with the formation of doublets $\left(3_{\gamma}^{+} 4_{\gamma}^{+}\right)$, $\left(5_{\gamma}^{+} 6_{\gamma}^{+}\right)$, etc., in contrast to the regular excitation pattern observed in experiment and reproduced by the collective Hamiltonian. This problem could be solved by including threebody boson terms in the IBM Hamiltonian, and work along this line is already in progress. When considering the calculated $B$ (E2) values for transitions in the ground-state band, the IBM reproduces the gradual decrease of transition rates with angular momentum for $L \geqslant 6$, reflecting the finiteness of the valence space. On the other hand, even though the collective 

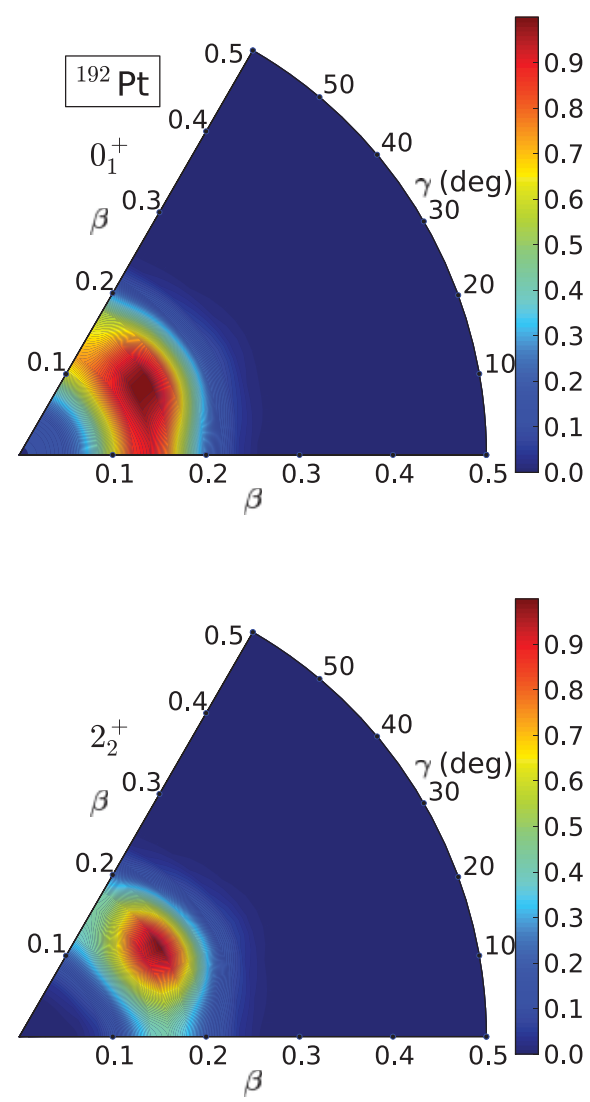
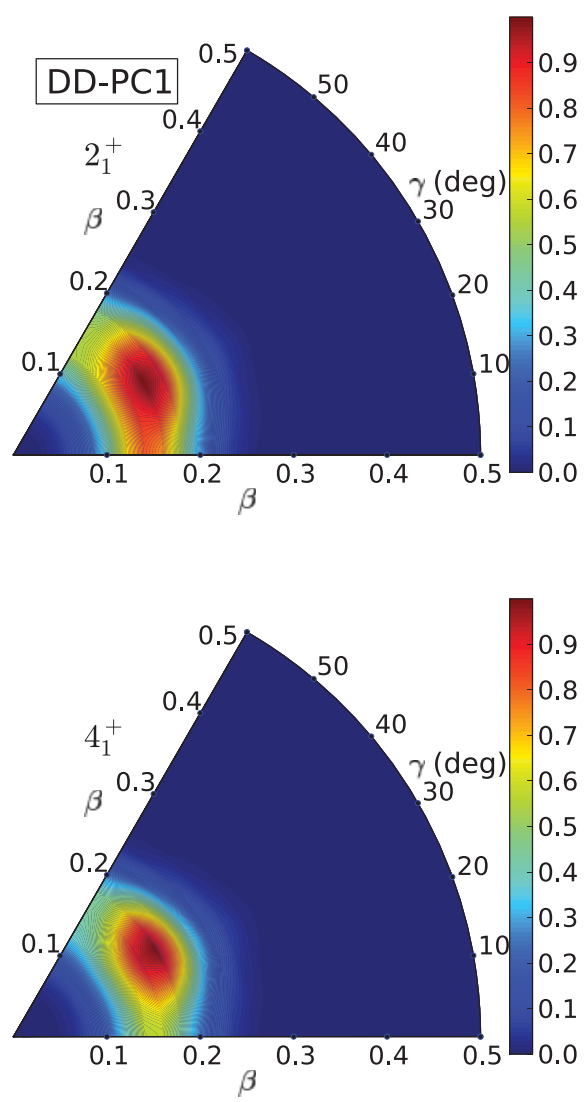

FIG. 4. (Color online) Same as described in the caption to Fig. 3, but for the eigenstates of the collective Hamiltonian.

Hamiltonian predicts parameter-free $B(\mathrm{E} 2)$ values in excellent agreement with experiment for transitions between low-spin states, the calculated transition probabilities keep increasing with angular momentum, in contrast to data.

Both models are based on binding energy surfaces calculated at zero rotational frequency. In general, this leads to effective rotational moments of inertia that are lower than empirical values, that is, the calculated rotational bands are stretched in energy compared to experimental bands. In the collective Hamiltonian, the moments of inertia can be improved by including the Thouless-Valatin dynamical rearrangement contributions. For the IBM Hamiltonian, one needs to include the kinetic rotational term [47] and perform the mapping of microscopic energy surfaces calculated at finite values of the rotational frequency. We have already started with the implementation of these modifications in our current version of the collective Hamiltonian based on relativistic EDF and in the IBM Hamiltonian. The comparison of the improved models will be the subject of a future study.

\section{ACKNOWLEDGMENTS}

This work has been supported in part by Grants-in-Aid for Scientific Research (A) 20244022 and No. 217368, and by MZOS, project No. 1191005-1010. K.N. and D.V. acknowledge support by the JSPS. T.N. acknowledges support by the Croatian Science Foundation.
[1] A. Bohr and B. R. Mottelson, Nuclear Structure (Benjamin, New York, 1969 and 1975), Vols. I and II.

[2] P. Ring and P. Schuck, The Nuclear Many-Body Problem (Springer, Berlin, 1980).

[3] M. Bender, P.-H. Heenen, and P.-G. Reinhard, Rev. Mod. Phys. 75, 121 (2003).

[4] P. Cejnar, J. Jolie, and R. F. Casten, Rev. Mod. Phys. 82, 2155 (2010).

[5] R. Rodríguez-Guzman, P. Sarriguren, L. M. Robledo, and S. Perez-Martin, Phys. Lett. B 691, 202 (2010).

[6] R. Rodríguez-Guzman, P. Sarriguren, and L. M. Robledo, Phys. Rev. C 82, 061302(R) (2010).
[7] T. Nikšić, D. Vretenar, G. A. Lalazissis, and P. Ring, Phys. Rev. Lett. 99, 092502 (2007).

[8] Z. P. Li, T. Nikšić, D. Vretenar, J. Meng, G. A. Lalazissis, and P. Ring, Phys. Rev. C 79, 054301 (2009).

[9] Z. P. Li, T. Nikšić, D. Vretenar, and J. Meng, Phys. Rev. C 81, 034316 (2010).

[10] T. H. R. Skyrme, Nucl. Phys. 9, 615 (1959).

[11] D. Vautherin and D. M. Brink, Phys. Rev. C 5, 626 (1972).

[12] J. Decharge, M. Girod, and D. Gogny, Phys. Lett. B 55, 361 (1975). 
[13] J. F. Berger, M. Girod, and D. Gogny, Nucl. Phys. A 428, 23c (1984).

[14] D. Vretenar, A. V. Afanasjev, G. A. Lalazissis, and P. Ring, Phys. Rep. 409, 101 (2005).

[15] T. Nikšić, D. Vretenar, and P. Ring, Prog. Part. Nucl. Phys. 66, 519 (2011).

[16] R. Rodríguez-Guzmán, J. L. Egido, and L. M. Robledo, Nucl. Phys. A 709, 201 (2002).

[17] T. R. Rodríguez and J. L. Egido, Phys. Rev. C 81, 064323 (2010).

[18] J. M. Yao, H. Mei, H. Chen, J. Meng, P. Ring, and D. Vretenar, Phys. Rev. C 83, 014308 (2011).

[19] P. Bonche, J. Dobaczewski, H. Flocard, P.-H. Heenen, and J. Meyer, Nucl. Phys. A 510, 466 (1990).

[20] J.-P. Delaroche, M. Girod, J. Libert, H. Goutte, S. Hilaire, S. Peru, N. Pillet, and G. F. Bertsch, Phys. Rev. C 81, 014303 (2010).

[21] T. Nikšić, Z. P. Li, D. Vretenar, L. Próchniak, J. Meng, and P. Ring, Phys. Rev. C 79, 034303 (2009).

[22] F. Iachello and A. Arima, The Interacting Boson Model (Cambridge University Press, Cambridge, UK, 1987).

[23] T. Otsuka, A. Arima, and F. Iachello, Nucl. Phys. A 309, 1 (1978).

[24] T. Otsuka, in Algebraic Approaches to Nuclear Structure, edited by R. F. Casten (Harwood, Chur, 1993), p. 195.

[25] T. Mizusaki and T. Otsuka, Prog. Theor. Phys. Suppl. 125, 97 (1996).

[26] K. Nomura, N. Shimizu, and T. Otsuka, Phys. Rev. Lett. 101, 142501 (2008).

[27] K. Nomura, N. Shimizu, and T. Otsuka, Phys. Rev. C 81, 044307 (2010).

[28] M. Bender, P. Bonche, T. Duguet, and P.-H. Heenen, Phys. Rev. C 69, 064303 (2004).

[29] T. Nikšić, D. Vretenar, and P. Ring, Phys. Rev. C 74, 064309 (2006).

[30] T. R. Rodríguez and J. L. Egido, Phys. Lett. B 663, 49 (2008).

[31] M. Bender and P.-H. Heenen, Phys. Rev. C 78, 024309 (2008).

[32] M. Anguiano, J. L. Egido, and L. M. Robledo, Nucl. Phys. A 696, 467 (2001).

[33] J. Dobaczewski, M. V. Stoitsov, W. Nazarewicz, and P.-G. Reinhard, Phys. Rev. C 76, 054315 (2007).
[34] D. Lacroix, T. Duguet, and M. Bender, Phys. Rev. C 79, 044318 (2009).

[35] M. Bender, T. Duguet, and D. Lacroix, Phys. Rev. C 79, 044319 (2009).

[36] T. Duguet, M. Bender, K. Bennaceur, D. Lacroix, and T. Lesinski, Phys. Rev. C 79, 044320 (2009).

[37] K. Nomura, T. Otsuka, R. Rodríguez-Guzmán, L. M. Robledo, and P. Sarriguren, Phys. Rev. C 83, 014309 (2011).

[38] K. Nomura, T. Otsuka, R. Rodríguez-Guzmán, L. M. Robledo, P. Sarriguren, P. H. Regan, P. D. Stevenson, and Zs. Podolyák, Phys. Rev. C 83, 054303 (2011).

[39] T. Nikšić, P. Ring, D. Vretenar, Y. Tian, and Z. Y. Ma, Phys. Rev. C 81, 054318 (2010).

[40] J. A. Cizewski, R. F. Casten, G. J. Smith, M. L. Stelts, W. R. Kane, H. G. Borner, and W. F. Davidson, Phys. Rev. Lett. 40, 167 (1978).

[41] R. F. Casten and J. A. Cizewski, Nucl. Phys. A 309, 477 (1978).

[42] T. Nikšić, D. Vretenar, and P. Ring, Phys. Rev. C 78, 034318 (2008).

[43] T. Nikšić, P. Ring, D. Vretenar, Y. Tian, and Z. Y. Ma, Phys. Rev. C 81, 054318 (2010).

[44] D. R. Inglis, Phys. Rev. 103, 1786 (1956).

[45] S. T. Belyaev, Nucl. Phys. 24, 322 (1961)

[46] A. E. L. Dieperink, O. Scholten, and F. Iachello, Phys. Rev. Lett. 44, 1747 (1980).

[47] K. Nomura, T. Otsuka, N. Shimizu, and L. Guo, Phys. Rev. C 83, 041302(R) (2011).

[48] L. M. Robledo, R. Rodríguez-Guzmán, and P. Sarriguren, J. Phys. G: Nucl. Part. Phys. 36, 115104 (2009).

[49] R. Rodriguez-Guzman, P. Sarriguren, L. M. Robledo, and J. E. García-Ramos, Phys. Rev. C 81, 024310 (2010).

[50] Brookhaven National Nuclear Data Center (NNDC) [http://www.nndc.bnl.gov/].

[51] C. M. Baglin, Nucl. Data Sheets 84, 717 (1998); B. Singh, ibid. 107, 1531 (2006); H. Xiaolong, ibid. 108, 1093 (2007).

[52] A. S. Davydov and G. F. Filippov, Nucl. Phys. 8, 237 (1958)

[53] K. Heyde, P. Van Isacker, M. Waroquier, and J. Moreau, Phys. Rev. C 29, 1420 (1984).

[54] R. F. Casten, P. von Brentano, K. Heyde, P. Van Isacker, and J. Jolie, Nucl. Phys. A 439, 289 (1985). 